\title{
A validation study of microscopy versus quantitative PCR for measuring Plasmodium falciparum parasitemia
}

\author{
Emma Ballard', Claire Y. T. Wang ${ }^{2}$, Tran Tinh Hien ${ }^{3}$, Nguyen Thanh Tong ${ }^{3}$, Louise Marquart ${ }^{1}$, Zuleima Pava', \\ Joel Tarning ${ }^{4,5}$, Peter O'Rourke ${ }^{1}$ and James S. McCarthy ${ }^{1,6^{*}}$ (D)
}

\begin{abstract}
Microscopy and $18 \mathrm{~S} \mathrm{qPCR}$ are the most common and field-friendly methods for quantifying malaria parasite density, and it is important that these methods can be interpreted as giving equivalent results. We compared results of quantitative measurement of Plasmodium falciparum parasitemia by microscopy and by $18 \mathrm{~S}$ qPCR in a phase 2 a study. Microscopy positive samples ( $n=355$; median 810 parasites/ $\mu \mathrm{L}$ [IQR 40-10,471]) showed close agreement with $18 \mathrm{~S} \mathrm{qPCR}$ in mean $\log _{10} / \mathrm{mL}$ transformed parasitemia values by paired $t$ test (difference $0.04,95 \% \mathrm{Cl}$ $-0.01-0.10, p=0.088$ ). Excellent intraclass correlation (0.97) and no evidence of systematic or proportional differences by Passing-Bablok regression were observed. $18 \mathrm{~S}$ qPCR appears to give equivalent parasitemia values to microscopy, which indicates $18 \mathrm{~S}$ qPCR is an appropriate alternative method to quantify parasitemia in clinical trials.
\end{abstract}

Keywords: Microscopy, qPCR, Plasmodium falciparum, Validation

\section{Background}

Microscopy is the gold standard method for identifying and quantifying malaria parasites [1]. Microscopists calculate parasitemia (parasites/microliter $(\mu \mathrm{L})$ of whole blood) as the parasites counted within a given number of microscope fields divided by the number of WBCs (thick film) or RBCs (thin film) in $\mu \mathrm{L}$ of whole blood multiplied by the total WBC (thick film) or RBC (thin film) counted within those fields [2]. The microscopy detection limit is generally $11-50$ parasites/ $\mu \mathrm{L}$ [3].

Nucleic acid amplification testing (NAAT) methods detect and quantify parasitemia with greater sensitivity and precision than microscopy, particularly for low levels of parasitemia [4]. Quantitative NAAT methods include quantitative PCR (qPCR) and quantitative reverse transcriptase PCR (qRT-PCR). NAAT methods for malaria target either specific DNA sequences of the parasite's genome, such as the gene encoding the $18 \mathrm{~S}$ ribosomal RNA gene (18S rDNA), or a constitutively transcribed RNA sequence such as $18 \mathrm{~S}$ rRNA of asexual parasites [1].

\footnotetext{
* Correspondence: james.mccarthy@qimrberghofer.edu.au

${ }^{1}$ QIMR Berghofer Medical Research Institute, Brisbane, Australia

${ }^{6}$ The University of Queensland, Brisbane, Australia

Full list of author information is available at the end of the article
}

The World Health Organization (WHO) has published standardised protocols for microscopy $[5,6]$. However, no equivalent protocols exist for NAAT. The Minimum Information for Publication of Quantitative Real-Time PCR Experiments (MIQE) guidelines [7] have led towards a more standardised approach to malaria NAAT [8, 9]. Murphy et al. [4] established the first formal external quality assurance (EQA) program to promote the reliability and comparability of results for NAAT across centres undertaking volunteer infection studies (VIS). In 2018, WHO published an operational manual for an ongoing EQA program for malaria NAAT [10].

Our aim was to compare microscopy and qPCR parasitemia values to demonstrate that qPCR is a suitable alternative to microscopy for quantifying parasitemia in clinical trials.

\section{Methods}

Participants with uncomplicated Plasmodium falciparum malaria were treated with a single dose of cipargamin (KAE609) in an open-label phase 2a study in Vietnam, as described by Hien et al. [11]. Twenty-five adult males had $6 \mathrm{~mL}$ of blood taken 4-6 hourly until they had two 
consecutive negative blood films, and subsequently once daily until day 8 .

Experienced microscopists followed standard procedures to count asexual parasites using both thick and thin blood films shortly after blood collection [6]. First, 200 WBCs were counted on thick films. If the parasite count was less than 10 after $200 \mathrm{WBCs}$, then up to 500 WBCs were counted. Thin films were used where the number of parasites exceeded 250 per 50 WBCs.

For the $18 \mathrm{~S}$ qPCR, DNA was extracted from a $200-\mu \mathrm{L}$ volume of packed RBCs using the QIAamp DNA blood mini kit (Qiagen). The Plasmodium-specific qPCR was performed on-site in real time using the protocol described by Imwong et al. [9] with primers and probes developed by Kamau et al. [12]. The method uses a hydrolysis probe real-time qPCR targeting the $18 \mathrm{~S}$ rRNA gene with a detection limit of 22 parasites/mL (SD 5). Samples were run in triplicate. Each $18 \mathrm{~S}$ qPCR run contained a positive and negative water control and internal phocine herpesvirus control. Cycle threshold values greater than 50 were considered non-detectable.

To account for possible confounding of results by circulating gametocytes, RNA was extracted from frozen packed RBCs using the QIAamp DNA blood mini kit (Qiagen) with DNase treatment and tested for the presence of the $P$. falciparum female gametocyte-specific mRNA $p f s 25$, using the primers, probe, and protocol described by Pasay et al. [13]. The ratio of $\log _{10}$ gametocytes/mL ( $p f s 25$ qRT-PCR) to $\log _{10}$ parasites/mL (18S qPCR) was then calculated for the 20 samples with both gametocyte and asexual parasite counts.

$\log _{10}$ parasitemia values using microscopy and $18 \mathrm{~S}$ qPCR were summarised by mean, standard deviation, coefficient of variation, median, interquartile range, and range. We used paired $t$ test, intraclass correlation coefficient (ICC), and Passing-Bablok regression to assess for agreement between $\log _{10}$ parasitemia values. ICC for consistency was calculated using a mixed effects model without interaction. The Passing-Bablok regression 95\% confidence interval (CI) was calculated with the bootstrap (quantile) method. Regression and residual plots were examined. The reference method was microscopy. All analyses were conducted in $\mathrm{R}$ Studio (version 1.0.136, $\mathrm{R}$ version 3.2.5).

\section{Results}

Of the 740 blood samples collected, 268 microscopy results were reported as non-detectable, 92 samples were clotted and unsuitable for $18 \mathrm{~S}$ qPCR, and 23 were missing values. Two $18 \mathrm{~S}$ qPCR assay results failed quality control checks, resulting in 355 matched samples across 22 participants. Median parasitemia value by microscopy was 810 parasites/ $\mu \mathrm{L}$ (IQR 40-10,471). $\log _{10}$ parasitemia values from microscopy were marginally lower (mean $5.81 \log _{10}$ units $/ \mathrm{mL}$ ) than values from 18S qPCR (mean $5.86 \log _{10}$ units $/ \mathrm{mL}$ ) (Table 1). Standard deviations and coefficients of variation were similar between methods. All samples with parasites detected by microscopy were also positive by $18 \mathrm{~S}$ qPCR.

The high ICC value indicated excellent consistency between microscopy and 18S qPCR (Table 2). The paired $t$ test indicated that $\log _{10}$ parasitemia values $/ \mathrm{mL}$ were not significantly different between microscopy and $18 \mathrm{~S}$ qPCR (mean difference 0.04 [95\% CI - 0.01-0.10], $p=0.088$ ). There was no evidence of a systematic or proportional bias by Passing-Bablok regression but a slight tendency towards non-linearity at lower levels of parasitemia (H statistic: $1.50 ; p<0.05$ ) (Fig. 1).

Our 18S qPCR assay was developed for clinical trials where the upper limit of quantification (ULoQ) is 250, 000 parasites/mL [9]. Half of the samples tested had parasitemia values above this ULoQ. We conducted sensitivity analysis to examine whether extrapolation of $18 \mathrm{~S}$ qPCR values above the ULoQ was reasonable $(n=155 \leq$ 250,000 parasites/mL, mean difference $0.07 \log _{10}$ units/ $\mathrm{mL}$ [95\% CI $-0.01-0.15, p=0.10]$ ), and found our conclusions unchanged, which indicates that extrapolation beyond the reference range was valid.

Across matched samples analysed by both $18 \mathrm{~S}$ qPCR and pfs 25 qRT-PCR assay the ratio of gametocytes to total parasites averaged 0.006 (SD 0.010) and ranged between 0.000 and 0.050 . Thus, our comparison of microscopy with $18 \mathrm{~S}$ qPCR was not confounded by the presence of gametocytes.

\section{Discussion}

Our findings support the use of the $18 \mathrm{~S}$ qPCR assay in clinical trials to quantify a wide range of parasitemia values, based on a strong agreement with microscopy. This has application to clinical trials in endemic settings (such as [11]) and to VIS in non-endemic settings.

Parasitemia values from microscopy and from $18 \mathrm{~S}$ qPCR showed good agreement and no evidence of systematic or proportional bias by Passing-Bablok regression, a method commonly used in clinical laboratory settings [14]. Our findings of a mean difference of 0.04

Table 1 Comparison of $\log _{10}$ parasitemia values $/ \mathrm{mL}$ by microscopy and $18 \mathrm{~S}$ qPCR

\begin{tabular}{lll}
\hline & Microscopy & 185 qPCR \\
\hline$n$ & 355 & 355 \\
Mean (SD) & $5.81(1.31)$ & $5.86(1.33)$ \\
CV\% & 22.5 & 22.8 \\
Median (IQR) & $5.91(4.60-7.02)$ & $6.00(4.65-7.00)$ \\
Range & $3.48-7.88$ & $2.30-8.18$ \\
\hline
\end{tabular}

CV\% coefficient of variation, IQR interquartile range, SD standard deviation 
Table 2 Agreement statistics for $\log _{10}$ parasitemia/mL by microscopy and $18 \mathrm{~S}$ qPCR

\begin{tabular}{ll}
\hline & Microscopy versus 18S qPCR \\
\hline ICC $(95 \% \mathrm{Cl})$ & 355 \\
Paired $t$ test $(95 \% \mathrm{Cl})$ & $0.965(0.956-0.971)$ \\
$\quad$ Mean difference $(95 \% \mathrm{Cl})$ & $0.04(-0.01-0.10)$ \\
$\quad p$ value & 0.088 \\
Passing-Bablok regression & \\
$\quad$ Intercept $(95 \% \mathrm{Cl})$ & $0.06(-0.18-0.28)$ \\
$\quad$ Slope $(95 \% \mathrm{Cl})$ & $1.01(0.97-1.04)$ \\
$\quad$ H statistic & 1.50
\end{tabular}

Cl confidence interval, ICC intraclass correlation coefficient

$\log _{10}$ units/mL are supported by several smaller studies. Nwakanma et al. [15] calculated a mean difference of $0.5 \log _{10}$ units (95\% CI - 1.4-0.4) for 81 thick films with values from an $18 \mathrm{~S}$ rRNA gene-targeted qPCR. Kamau et al. [16] reported a much larger mean difference of $0.87 \log _{10}$ units for 60 samples, although they used averaged thick or thin film counts using a multiplex qPCR containing an $18 \mathrm{~S}$ rRNA gene target.

Although microscopy is the gold standard for quantifying parasitemia, microscopy cannot quantify parasitemia at low levels and its accuracy is influenced by the film type (thick or thin), microscopist's expertise, and WBC count (theoretical or actual) used [2]. Quantification of sexual lifecycle stages may not be included in microscopy counts, such as in this study. However, we did not find evidence of over-estimation by $18 \mathrm{~S}$ qPCR due to low numbers of gametocytes in matched samples. If deconvolution of asexual parasitemia versus gametocytemia is required, then inclusion of qRT-PCR assays targeting specific life cycle stages is recommended [13].

18S qPCR assays cannot discern whether target DNA is within a viable or dead parasite, or elsewhere, such as cell-free DNA. Parasite counts from 18S qPCR assays could be influenced by cell-free Plasmodium DNA in the saliva, blood, or stool of malaria-infected humans [17]. This could lead to overestimation of intact and viable parasites if parasites from target DNA elsewhere are counted [18]. However, the agreement between the

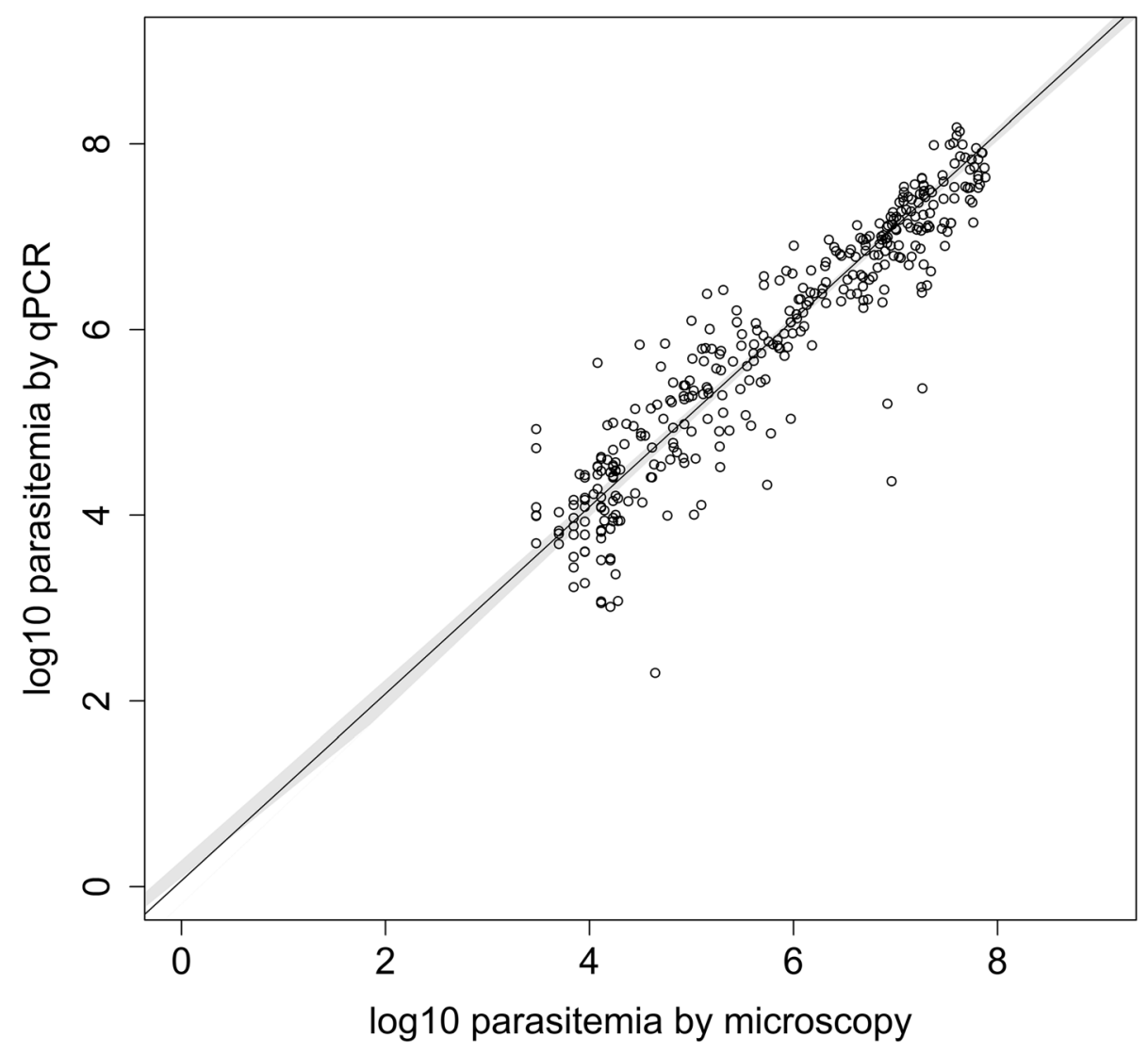

Fig. 1 Passing-Bablok regression fit comparing microscopy and 18S qPCR. The plot shows $\log _{10}$ parasitemia by microscopy and $18 \mathrm{~S}$ qPCR. The solid black line represents the fitted Passing-Bablok regression line. The $95 \%$ confidence bounds, in grey, were calculated using the bootstrap quantile method 
parasitemia values estimated by microscopy and $18 \mathrm{~S}$ qPCR in our study (patients treated with cipargamin) suggests confounding due to cell-free DNA is unlikely.

\section{Conclusions}

In this study, we compared the most common and fieldfriendly methods for parasite quantification. Although other methods, including flow cytometry [19] and droplet digital PCR [20], can quantify parasitemia without a standard curve, they have high operational costs and are unlikely to be realistic options for many laboratories. In conclusion, our results support the use of $18 \mathrm{~S}$ qPCR to give equivalent estimates to those from microscopy for $P$. falciparum parasitemia in clinical trials, particularly drug studies where accurate quantification is required across a wide range of parasitemia.

\section{Abbreviations}

Cl: Confidence interval; EQA: External quality assurance; ICC: Intraclass correlation coefficient; IQR: Interquartile range; MIQE: Minimum Information for Publication of Quantitative Real-Time PCR Experiments; NAAT: Nucleic acid amplification testing; qPCR: Quantitative polymerase chain reaction; qRT-PCR: Quantitative reverse transcriptase polymerase chain reaction; RBC: Red blood cell; SD: Standard deviation; ULOQ: Upper limit of quantification; VIS: Volunteer infection study; WBC: White blood cell; WHO: World Health Organization

\section{Acknowledgements}

We thank the participants of the original published study. We acknowledge Jacinda Wilson for assistance with manuscript submission.

\section{Authors' contributions}

$T T H, N T T$, and JT conducted the original study and supplied the data. JSM, $P O, E B$, and LM conceptualised and generated the idea for the study. EB conducted overall data analysis. EB, CW, ZP, PO, and JSM drafted the manuscript. All authors read and approved the final manuscript.

\section{Funding}

The $18 \mathrm{~S}$ and pfs 25 assays were funded by Novartis Pharma AG. The original published study was part of the Wellcome Trust Tropical Medicine Research Programmes in Vietnam and Thailand and was supported by Novartis Pharma AG.

\section{Availability of data and materials}

The datasets analysed during the current study are available from the corresponding author on reasonable request.

\section{Ethics approval and consent to participate}

Ethical approval and consent to participate is documented in the publication of the original study. The original published study was registered in the ClinicalTrials.gov database under identifier NCT01836458.

\section{Consent for publication}

Not applicable.

\section{Competing interests}

The authors declare that they have no competing interests.

\section{Author details}

${ }^{1}$ QIMR Berghofer Medical Research Institute, Brisbane, Australia. ${ }^{2}$ Queensland Paediatric Infectious Diseases Laboratory, Centre for Children's Health Research, Brisbane, Australia. ${ }^{3}$ Oxford University Clinical Research Unit-Hospital for Tropical Diseases, Ho Chi Minh City, Vietnam. ${ }^{4}$ Mahidol-Oxford Tropical Medicine Research Unit, Faculty of Tropical Medicine, Mahidol University, Bangkok, Thailand. ${ }^{5}$ Centre for Tropical Medicine and Global Health, Nuffield Department of Medicine, Oxford University, Oxford, UK. ${ }^{6}$ The University of Queensland, Brisbane, Australia.
Received: 16 June 2019 Accepted: 12 August 2019

Published online: 27 August 2019

\section{References}

1. Murphy SC, Shott JP, Parikh S, Etter P, Prescott WR, Stewart VA. Malaria diagnostics in clinical trials. Am J Trop Med Hyg. 2013;89(5):824-39.

2. O'Meara W, McKenzie F, Magill A, Forney J, Permpanich B, Lucas C, et al. Sources of variability in determining malaria parasite density by microscopy. Am J Trop Med Hyg. 2005;73(3):593-8.

3. Kobayashi T, Gamboa D, Ndiaye D, Cui L, Sutton PL, Vinetz JM. Malaria diagnosis across the international centers of excellence for malaria research: platforms, performance, and standardization. Am J Trop Med Hyg. 2015;93(3 Suppl):99-109.

4. Murphy SC, Hermsen CC, Douglas AD, Edwards NJ, Petersen I, Fahle GA, et al. External quality assurance of malaria nucleic acid testing for clinical trials and eradication surveillance. PLoS One. 2014;9(5):e97398.

5. World Health Organization. Malaria microscopy quality assurance manual (version 2); 2016. https:/www.who.int/malaria/publications/atoz/9789241549394/en/.

6. World Health Organization \& UNICEF/UNDP/World Bank/WHO Special Programme for Research and Training in Tropical Diseases. Microscopy for the detection, identification and quantification of malaria parasites on stained thick and thin blood films in research settings: procedure: methods manual (version 1.0); 2015. https://apps.who.int/iris/handle/10665/163782.

7. Bustin SA, Benes V, Garson JA, Hellemans J, Huggett J, Kubista M, et al. The MIQE guidelines: minimum information for publication of quantitative realtime PCR experiments. Clin Chem. 2009;55(4):611-22.

8. Alemayehu S, Feghali KC, Cowden J, Komisar J, Ockenhouse CF, Kamau E. Comparative evaluation of published real-time PCR assays for the detection of malaria following MIQE guidelines. Malar J. 2013;12(1):277.

9. Imwong M, Hanchana S, Malleret B, Renia L, Day N, Dondorp A, et al. Highthroughput ultrasensitive molecular techniques for quantifying low-density malaria parasitemias. J Clin Microbiol. 2014;52(9):3303.

10. World Health Organization. WHO external quality assurance scheme for malaria nucleic acid amplification testing: operation manual (version 1.0); 2018. Licence: CC BY-NC-SA 3.0 IGO. https:/apps.who.int/iris/handle/10665/259950.

11. Hien TT, White NJ, Thuy-Nhien NT, Hoa NT, Thuan PD, Tarning J, et al. Estimation of the in vivo MIC of cipargamin in uncomplicated Plasmodium falciparum malaria. Antimicrob Agents Chemother. 2017;61:2.

12. Kamau E, Tolbert LS, Kortepeter L, Pratt M, Nyakoe N, Muringo L, et al. Development of a highly sensitive genus-specific quantitative reverse transcriptase real-time PCR assay for detection and quantitation of plasmodium by amplifying RNA and DNA of the $18 \mathrm{~S}$ rRNA genes. J Clin Microbiol. 2011;49(8):2946-53.

13. Pasay CJ, Rockett R, Sekuloski S, Griffin P, Marquart L, Peatey C, et al. Piperaquine monotherapy of drug-susceptible Plasmodium falciparum infection results in rapid clearance of parasitemia but is followed by the appearance of gametocytemia. J Infect Dis. 2016;214(1):105-13.

14. Pant V, Tumbapo A, Karki B. Inter-instrumental comparison for the measurement of electrolytes in patients admitted to the intensive care unit. Int J Gen Med. 2017;10:145-9.

15. Nwakanma DC, Gomez-Escobar N, Walther M, Crozier S, Dubovsky F, Malkin E, et al. Quantitative detection of Plasmodium falciparum DNA in saliva, blood, and urine. J Infect Dis. 2009;199(11):1567-74.

16. Kamau E, Alemayehu S, Feghali KC, Saunders D, Ockenhouse CF. Multiplex qPCR for detection and absolute quantification of malaria. PLoS One. 2013;8(8):e71539.

17. Weerakoon KG, McManus DP. Cell-free DNA as a diagnostic tool for human parasitic infections. Trends Parasitol. 2016;32(5):378-91.

18. Vafa Homann M, Emami SN, Yman V, Stenstrom C, Sonden K, Ramstrom H, et al. Detection of malaria parasites after treatment in travelers: a 12-months longitudinal study and statistical modelling analysis. EBioMedicine. 2017;25:66-72.

19. Campo JJ, Aponte JJ, Nhabomba AJ, Sacarlal J, Angulo-Barturen I, JimenezDiaz MB, et al. Feasibility of flow cytometry for measurements of Plasmodium falciparum parasite burden in studies in areas of malaria endemicity by use of bidimensional assessment of YOYO- 1 and autofluorescence. J Clin Microbiol. 2011;49(3):968-74.

20. Koepfli C, Nguitragool W, Hofmann NE, Robinson L, Ome-Kaius M, Sattabongkot J, et al. Sensitive and accurate quantification of human malaria parasites using droplet digital PCR (ddPCR). Sci Rep. 2016;6:39183.

\section{Publisher's Note}

Springer Nature remains neutral with regard to jurisdictional claims in published maps and institutional affiliations. 